\title{
Charge Collection Mechanism in MEMS Capacitive Switches
}

\author{
Matroni Koutsoureli, Loukas Michalas and George Papaioannou \\ University of Athens \\ Physics Dpt., Solid State Physics Section \\ Athens, Greece \\ mkoutsoureli@phys.uoa.gr
}

\begin{abstract}
The present paper investigates the effect of stressing bias magnitude and stressing time on the discharging process in MEMS capacitive switches. The calculation of discharge current through the dielectric film is based on monitoring the rate of shift of bias for up-state minimum capacitance. The data analysis shows that the discharge current lies in the range of femtoAmperes and the calculated discharge time constant depends directly on the time window of observation and on the stressing conditions. Moreover the analysis reveals an increase of trapped charge that remains in the bulk of the dielectric film for very long time as the stressing bias increases. The dominant discharge process, taking place under an intrinsic field of about $10^{3} \mathrm{~V} / \mathrm{cm}$, is found to be the hopping effect.
\end{abstract}

Keywords-component; Dielectric charging; MEMS capacitive switches; reliability

\section{INTRODUCTION}

The effect of dielectric charging constitutes a major reliability issue in MEMS capacitive switches. In spite of the intensive research during last decade the effect is still not fully understood. The presence of high electric field during actuation gives rise to charge injection that in turn shifts the capacitance voltage characteristic [1] and limits the device lifetime, the latter depending significantly on the actuation voltage magnitude [2]. In a cycling device the time to failure is determined by the charging and discharging, the later through the bottom electrode, and the time during pull-in and pull-up state respectively. In order to balance these processes various actuation bias profiles [2] or adjustable duty cycles [3] have been proposed.

The lifetime of a capacitive switch is directly determined by the competition between the charging process, which occurs under high electric fields, and the discharging one, which occurs under the presence of low intrinsic fields that are generated by the non-uniform distribution of the injected charge [4]. The charging process has been investigated in MEMS switches [5] and bare silicon nitride films [6] and in both cases it was found that macroscopically follows power law relation. The discharge process through the dielectric film has been investigated by Kelvin Probe Force Microscopy (KPFM) method, applied on both silicon nitride dielectric films for MEMS and on the top electrode potential of MetalInsulator-Metal (MIM) capacitors [7], as well as by the shift of the bias for minimum capacitance of MEMS capacitive switches $[8,9]$. Moreover, the discharge current transient has been directly recorded for a time window of observation of a few hundred seconds [10] and calculated for a window in excess of $10^{4} \mathrm{sec}$ [9], the latter leading to a better approximation of the total stored charge dissipation. In all cases it was reported that macroscopically it follows the stretched exponential law. This behavior has been attributed to the fact that the charge collection is complex because the dielectric film is deposited at low temperatures, fact that leads to highly disordered materials, which deviate from stoichiometry and contain large concentration of defects $[11,12]$. Finally, the investigation of temperature effect on the dielectric charging in silicon nitride based MEMS has revealed that the process is thermally activated and attributed to hopping in a continuum of trap states, where the hopping is expected to be variable rather than nearest neighbor [13].

The aim of the present work is to exploit the possibility of monitoring the long time discharge current in order to investigate the effect of stress bias level and stressing time on the discharge process in MEMS capacitive switches and provide a better understanding of the responsible mechanism. The discharge process takes place under the presence of intrinsic field in the range of $10^{3}-10^{4} \mathrm{~V} / \mathrm{cm}$, a fact that has not been taken into account up to now. Finally, it is shown that stressing time plays a key issue role on the discharge process.

\section{THEORETICAL BACKGROUND}

\section{A. Discharge in MEMScapacitive switches}

In order to determine the discharge current transient in a MEMS capacitive switch we adopt the device model proposed in [14] which includes a fixed non-flat metal plate of area $A$ covered with a dielectric film of uniform thickness $d_{\varepsilon}$, dielectric constant $\varepsilon_{r}$ and a volume charge density $\psi(x, y, z)$.

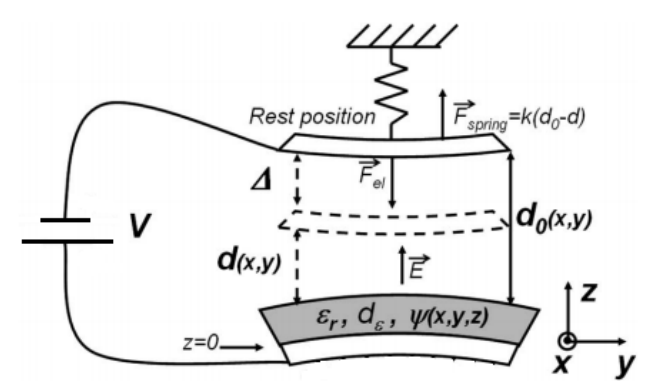

Fig. 1: Model of a capacitive switch with non-uniform trapped charge and air gap distributions [14]. 
Above it a rigid but non-flat moveable metal plate is fastened with a linear spring $k$ to a fixed wall above the dielectric layer at a rest position $d_{0}(x, y)$, as shown in Fig. 1. According to [14] the electrostatic force $F_{e l}$ exerted to the top plate and resulting to a displacement $\Delta$ when a dc bias source of amplitude $V$ is applied to the two plates can be expressed in a compact form of:

$$
F_{e l}(\Delta)=\frac{A}{2 \varepsilon_{0}}\left[\left(V \mu_{\alpha}-\mu_{\beta}\right)^{2}+V^{2} \sigma_{\alpha}^{2}+\sigma_{\beta}^{2}-2 V \operatorname{cov}(\alpha, \beta)\right]
$$

where $\mu, \sigma^{2}$, and cov denote the mean, variance, and covariance, respectively, of the capacitance $\alpha(x, y, \Delta)$ and charge $\beta(x, y, \Delta)$ density distributions. The distributions of capacitance per unit area and charge density induced at armature area are respectively:

$$
\begin{aligned}
& \alpha(x, y, \Delta)=\frac{\varepsilon_{0}}{\left[d_{0}(x, y)-\Delta\right]-\left(d_{\varepsilon} / \varepsilon_{r}\right)} \\
& \beta(x, y, \Delta)=\frac{d_{\varepsilon}}{\varepsilon_{r} \varepsilon_{0}} \cdot \psi_{e q}(x, y) \cdot \alpha(x, y)
\end{aligned}
$$

where $\psi_{e q}(x, y)$ denotes the equivalent surface charge density distribution.

In the general case of distributed equivalent charge $[\psi(x, y, z)]$ and air gap $\left[d_{0}(x, y)\right]$, Eq. 1 cannot be further simplified. The bias at which the up-state capacitance attains minimum $\left(V_{\min }\right)$ is the bias for which the electrostatic force becomes minimum independently of the charge and air gap distributions and it is given by:

$$
V_{\min }=\frac{\mu_{\alpha} \mu_{\beta}+\operatorname{cov}(\alpha, \beta)}{\mu_{\alpha}^{2}+\sigma_{\alpha}^{2}}
$$

The assessment of a MEMS capacitive switch, in the pullup state, is based on the analysis of capacitance-voltage characteristic, which provides information on both the shift of the bias for minimum capacitance and the vitiation of the magnitude of the minimum capacitance, both with time. Further, if for the sake of simplicity it is assumed that the capacitance variance is low then Eq. 3 simplifies to:

$$
V_{\min }=\frac{\mu_{\beta}}{\mu_{\alpha}}=\frac{d_{\varepsilon}}{\varepsilon_{r} \varepsilon_{0}} \cdot \mu_{\psi}
$$

where $\mu_{\psi}$ represents the mean value of the charge distribution projected at dielectric film surface $\left[\psi_{e q}(x, y)\right]$. In order to proceed it is necessary to emphasize that the impact of the minimum electrostatic force on the measured capacitance, at minimum of $\mathrm{C}-\mathrm{V}$ characteristic, is mitigated by creep, which increases the value of $\mathrm{C}_{\min }$. Obviously this introduces an important drawback that allows the exploitation of the time evolution of charge distribution variance with extreme care. Such problem can be practically dealt only with carefully designed test structures. Taking all these into account the calculated net discharge current density is given by:

$$
J_{\text {disch }}(t)=-\frac{d \mu_{\psi}(t)}{d t}=-\frac{\varepsilon_{r} \varepsilon_{0}}{d_{\varepsilon}} \cdot \frac{d V_{\min }(t)}{d t}
$$

which describes the average value of discharge current density due to charge collection by the bottom electrode.
Finally, taking into account that the injected charges are confined near the injecting electrodes $[4,8]$ we can assume that the intrinsic electric field can be approximated by: $F=\frac{V_{\min }}{d_{\varepsilon}}$. Then the corresponding conductivity at time t, during the discharge process, is given by:

$$
\sigma(t)=\frac{\varepsilon_{s} \varepsilon_{0}}{V_{\min }(t)} \cdot\left|\frac{d V_{\min }(t)}{d t}\right|
$$

\section{B. Hopping conductivity}

According to a traditional approach to the analysis of charge carrier kinetics in disordered hopping systems, the carrier jump rate $v$ from a starting site of energy $E_{s}$ to a target site of energy $E_{t}$ over the distance $r$ is $[15,16]$ :

$$
v=v_{0} \cdot \exp (-u)
$$

where $\mathrm{u}$ is a hopping parameter defined as:

$$
u\left(E_{s}, E_{t}, r\right)=2 \gamma r+ \begin{cases}0, & E_{t}<E_{s}+e F r z \\ \frac{E_{t}-E_{s}-e F r z}{k_{B} T}, & E_{t}>E_{s}+e F r z\end{cases}
$$

Here, $\mathrm{F}$ is the intensity of the electric field, $\mathrm{T}$ is temperature, $v_{0}$ the attempt to jump frequency, $\gamma$ the inverse localization radius, e the elementary charge, $\mathrm{k}_{\mathrm{B}}$ is the Boltzmann's constant and $\mathrm{z}=\cos \theta$ with $\theta$ being the angle between the field and the jump direction. The hopping parameter clearly shows that the jump rate is determined by the presence and magnitude of electric field intensity, but only if the latter is strong enough in order to significantly change the energy difference between starting and target sites. Moreover the distribution of trapping states in the band gap plays a key issue role on the application of Eq. 8.

The field effect on the variable range hopping was considered by Shklovskii [17] who showed that electric field plays a role similar to temperature. In order to obtain the field dependence of the conductivity $\sigma(\mathrm{F})$ at high fields, the temperature $\mathrm{T}$ in the well-known dependence $\sigma(\mathrm{T})$ for low fields can be replaced by a function $\mathrm{T}_{\mathrm{eff}}$ of the form:

$$
k_{B} T_{e f f}=\frac{e F}{2 \gamma}
$$

Extending the percolation theory of hopping conduction beyond the limit of linear response and including the effect of local chemical potential, Pollak and Riess [18] have shown that in the regime of moderate fields (smaller than about $10^{5} \mathrm{~V} / \mathrm{cm}$ at $300 \mathrm{~K})$, where the condition $\mathrm{eFr}_{\mathrm{m}}>\mathrm{k}_{\mathrm{B}} \mathrm{T}$ holds, the hopping conductivity $\sigma(\mathrm{F})=\mathrm{J}(\mathrm{F}) / \mathrm{F}$ obeys a relationship of the form:

$$
\sigma(F)=\sigma(0) \cdot \exp \left[\frac{e \cdot F \cdot l}{k_{B} \cdot T}\right]
$$

Here, $\mathrm{F}$ is the intensity of the electric field, $\mathrm{J}$ the current density, e the electron charge, $1=\mathrm{Cr}_{\mathrm{m}}$ the hopping length, $\mathrm{r}_{\mathrm{m}}$ the maximum hopping length on the percolation paths, $\mathrm{k}_{\mathrm{B}}$ Boltzmann's constant, $\mathrm{T}$ is the temperature and $\mathrm{C}$ is a constant which is found to be equal to 0.17 and 0.18 for the case of three and two dimensions respectively. 


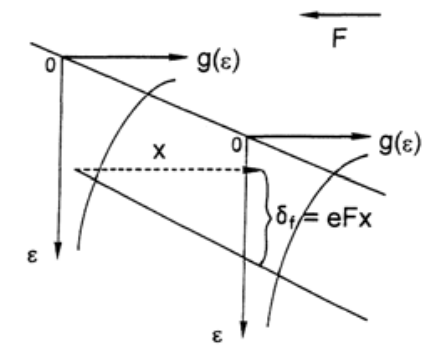

Fig. 2: Electron hop over a distance $\mathrm{x}$ in the case of high electric field $\mathrm{F}$ $\lceil 17\rceil$

\section{EXPERIMENTAL PROCEDURE}

The switches measured are bridge-type capacitive switches with $250 \mathrm{~nm}$ thick $\mathrm{Si}_{3} \mathrm{~N}_{4}$ dielectric film. The membrane is suspended about $2.2 \mu \mathrm{m}$ above the dielectric in the unactuated position forming a $150 \mu \mathrm{m} \times 80 \mu \mathrm{m}$ capacitor which shunts the RF signal to ground in the pull-in state. The devices were stressed for different times with bias levels ranging from $\mathrm{V}_{\mathrm{pi}}$ to $1.6 \mathrm{~V}_{\mathrm{pi}}$ while the pull-in voltage was $\mathrm{V}_{\mathrm{pi}}=20 \mathrm{~V}$. After each stress cycle the devices were assessed by obtaining the $\mathrm{C}-\mathrm{V}$ characteristics in the up state for $10000 \mathrm{~s}$ (Fig. 3) in order to monitor the shift of the bias for minimum capacitance towards the pre-stress level. The bias for up state capacitance minimum was determined by fitting a parabola to the experimental data, assuming a very small capacitance variance in order to take advantage of Eqs. 4 and 5.

All measurements have been performed at room temperature and in vacuum in order to avoid any interference from humidity [19].

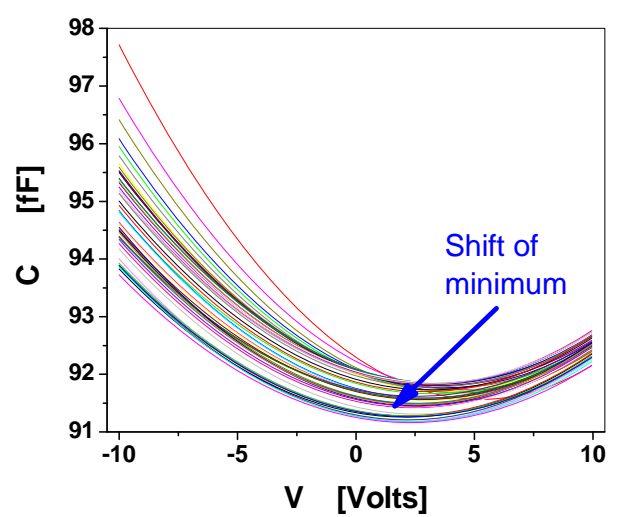

Fig. 3: Evolution of pull-up state capacitance-voltage characteristic of a switch after being stressed for $5 \mathrm{~min}$ at $30 \mathrm{~V}$.

\section{RESULTS AND DISCUSSION}

The shift of bias for minimum capacitance (Fig. 4) after stressing the devices at different bias levels clearly shows an increase of potential offset $\left(\mathrm{V}_{\text {offset }}\right)$ during the time window of the present experiment, arising from charges which are collected under very long time constants, as the stressing bias increases. This shows that only a fraction of stored charge is collected within the selected time window of observation $\left(10^{4}\right.$ s) while more and more charges with very long relaxation

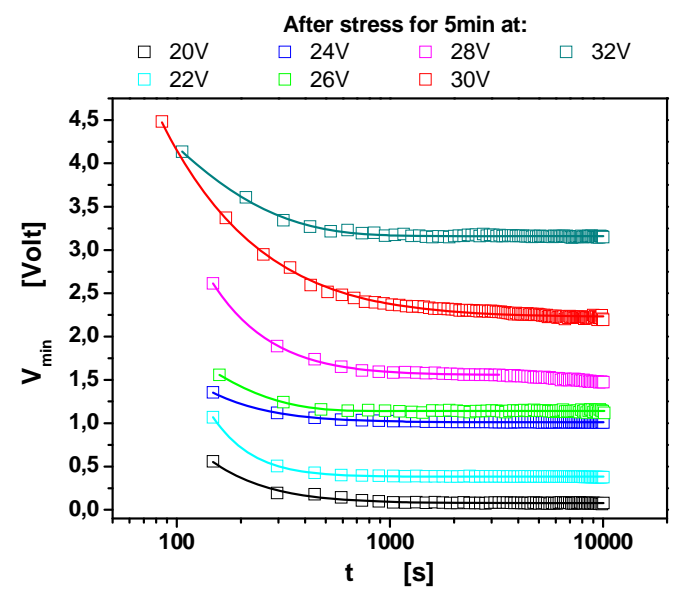

Fig. 4: Transients of $\mathrm{V}_{\min }$ after stressing the devices for $5 \mathrm{~min}$ at different bias levels.

times remain in the bulk of the dielectric as the stressing bias increases.

Moreover, taking into account that the dielectric film thickness is $250 \mathrm{~nm}$, the discharge process takes place under an intrinsic electric field in the range $1-10 \times 10^{3} \mathrm{~V} / \mathrm{cm}$, a fact that indicates that the Poole-Frenkel effect does not contribute and the dominant mechanism during discharge is expected to be the hopping effect [20].

The transients of $\mathrm{V}_{\min }$ after stressing the devices at $30 \mathrm{~V}$ for different stressing times have shown an increase of relaxation times, as it is shown in Fig. 5. In a previous investigation [4] it has been shown that an increase of stressing time on the dielectric film of MEMS capacitive switches results to an increase of trapped charge. In addition, the trapped charge centroid is shifting deeper into the dielectric film, as the stressing time increases.

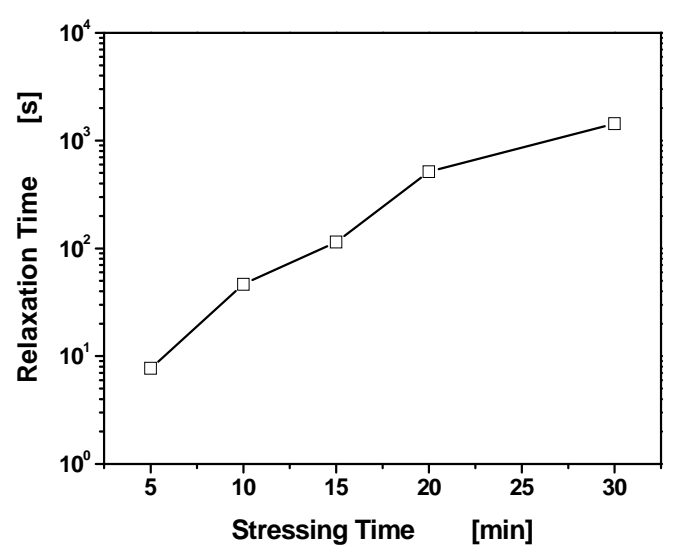

Fig. 5: Relaxation time for the decay of $\mathrm{V}_{\text {min }}$, after stressing the devices at $30 \mathrm{~V}$ for different stressing times.

In silicon rich hydrogenated silicon nitride metal semiconductor - metal capacitors the photon-emission experiments have shown the existence of nonthermal (hot) electrons under strong applied electric fields [21]. The 
breaking of $\mathrm{Si}-\mathrm{H}$ bonds from these hot electrons free $\mathrm{H}$ atoms that move further to break weak $\mathrm{Si}-\mathrm{Si}$ bonds, thus generating additional deep trap states in the bulk of the dielectric. Moreover, it has been reported that adding charge to an insulating system takes it out of thermal equilibrium and that conductivity in hopping systems may relax to an equilibrium value with extremely long time periods that are many orders of magnitude larger than the Maxwell time [22]. This slow equilibration process of the electronic system is ascribed to the inhomogeneous nature of charge transport and to the slow energy relaxation which are inherent features of disordered materials.

According to all these, it becomes apparent that the increase of stressing time results to generation of deep defect states with large relaxation times. This phenomenon is even pronounced when the stressing field is increased. In this case the defect states that are created during stress introduce a significant offset to the transients of $\mathrm{V}_{\min }$. The increase of the stressing field introduces deeper trap states in the bulk of the dielectric with relaxation times greater than the time window of observation.

The calculated discharge current is found to be in the range of femto-Amperes (Fig. 6) and for the present devices vanishes after a time of about $1000 \mathrm{~s}$. The total charge density per unit area that will be collected at infinite time can also be calculated by integrating the discharge current density:

$$
\rho_{\text {total }}=\int_{0}^{\infty} J_{\text {disch }}(t) \cdot d t
$$

and as it is shown from the inset graph of Fig. 6 it increases with the intensity of the stressing field. This has also been reported for the discharge process in Metal - Insulator - Metal structures, obtained from TSDC assessment [23].

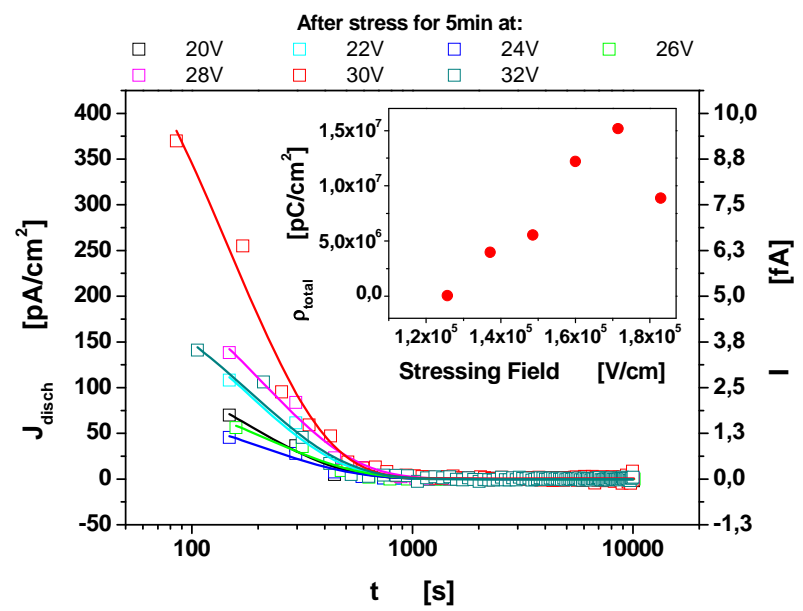

Fig. 6: Calculated discharge currents after stressing the devices for $5 \mathrm{~min}$ at different bias levels. The inset graph shows the total charge density per unit area that will be collected at infinite time.

As already mentioned the value of $\mathrm{V}_{\min }$ corresponds to the value of the applied bias at which the electrostatic force between the moving armature of the device and the surface of the dielectric film is minimized (almost zero). Thus $\mathrm{V}_{\min }$ should also be equal to the average potential at the surface of the

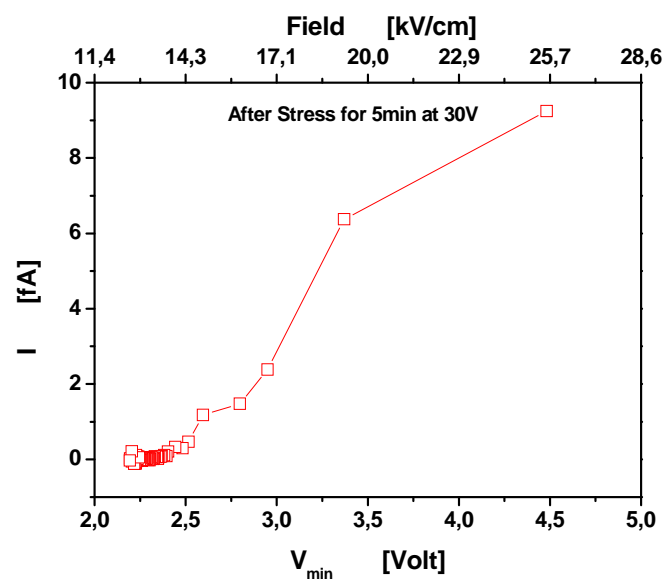

Fig. 7: Current - Voltage characteristic during discharge after stressing the device for $5 \mathrm{~min}$ at $30 \mathrm{~V}$.

dielectric. Having monitored the value of $\mathrm{V}_{\min }$ and calculated the discharge current enables us to monitor both the current voltage (I-V) characteristic and the corresponding conductivity as the discharge process proceeds with time.

Fig. 7 shows the I-V characteristic for a device after being stressed for $5 \mathrm{~min}$ at $30 \mathrm{~V}$. Moreover, the dependence of the conductivity on the average intrinsic electric field is obtained and shown on Fig. 8.

Govor et al. have shown [24] that at very low values of the electric field (in the range of $\mathrm{V} / \mathrm{cm}$ ) there is linear dependence between the hopping conductivity and the electrical field strength (ohmic region). With increasing electrical field strength, the regime of ohmic conductivity passes over to an interval with a stronger rise in conductivity while in the high electrical field regime there is an apparent saturation of conductivity. So, at constant temperature the conductivity can be expressed as:

$$
\sigma(E)=\sigma(0) \cdot \exp \left[A \cdot E^{n}\right]
$$

Exponent $\mathrm{n}$ equals to unity within the low electrical field range and Eq. 12 becomes identical to Eq. 10. At higher fields $n$ becomes zero (saturation) and then it is found to be negative for even stronger electric fields [24].

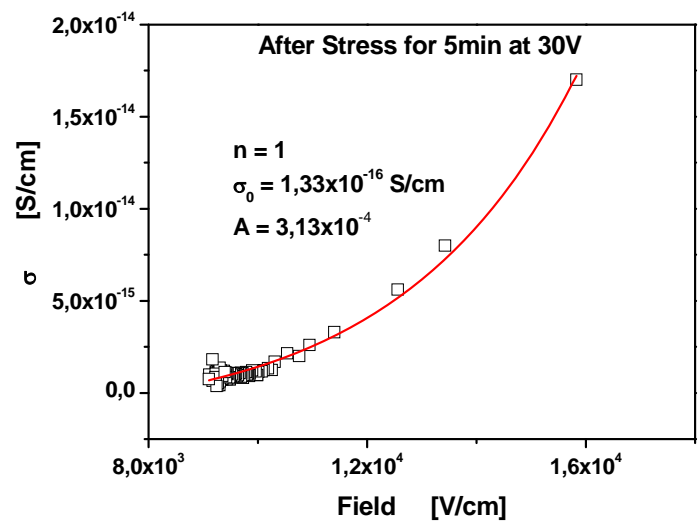

Fig. 8: Conductivity versus the intensity of electric field during discharge after stress for $5 \mathrm{~min}$ at $30 \mathrm{~V}$. The red line corresponds to the fit of data with Eq. 12. Values of fitted parameters are also shown. 
In our case, we cannot observe the low and high electrical field regions but only the moderate field range. So, in moderate fields the conductivity is found to increase exponentially with the intensity of the electric field (Fig. 8).

\section{CONCLUSIONS}

The shift of the bias for the minimum up - state capacitance and the dielectric film thickness are used to calculate the discharge current in the bulk of the dielectric film of MEMS capacitive switches. The decay of bias for minimum up - state capacitance has been recorded for $10^{4} \mathrm{~s}$ and the determined discharge current is found to be in the range of femto-Amperes. The discharging process has been examined for different stress conditions. The increase of stressing time and stressing field result to generation of deep trap states in the bulk of the dielectric with very long time constants (greater than the time window of observation). Moreover it has been presented that the discharge takes place under an intrinsic field in the range of $10^{3}-10^{4} \mathrm{~V} / \mathrm{cm}$ and hopping is the dominant conduction mechanism. Conductivity during discharge has been also found to depend exponentially from the electric field, in agreement to hopping systems.

\section{ACKNOWLEDGEMENT}

This research has been co-financed by the European Union (European Social Fund - ESF) and Greek national funds through the Operational Program "Education and Lifelong Learning" of the National Strategic Reference Framework (NSRF) - Research Funding Program: "Heracleitus II Investing in knowledge society through the European Social Fund".

The authors would also like to acknowledge the support from ENIAC-2010 NANOCOM and FP7 NANOTEC (GA No: 288531) projects.

\section{REFERENCES}

[1] J. Wibbeler, G. Pfeifer and M. Hietschold, "Parasitic charging of dielectric surfaces in capacitive microelectromechanical systems (MEMS)", Sensors and Actuators A, vol. 71, pp 74-80, 1998.

[2] C. Goldsmith, J. Ehmke, A. Malczewski, B. Pillans, S. Eshelman, Z. Yao, J. Brank, and M. Eberly, "Lifetime Characterization of Capacitive RF MEMS Switches”, IEEE MTT-S Digest pp. 227-230, 2001.

[3] H. Yamazaki, T. Ikehashi, T. Ohguro, E. Ogawa, K. Kojima, K. Ishimaru and H. Ishiuchi, "An intelligent bipolar actuation method with high stiction immunity for RF MEMS capacitive switches and variable capacitors", Sensors and Actuators A, vol. 139, pp. 233-236, 2007.

[4] G. Papaioannou, F. Coccetti and R. Plana, "On the modeling of dielectric charging in RF-MEMS capacitive switches", 2010 Topical Meeting on Silicon Monolithic Integrated Circuits in RF Systems (SiRF), N. Orleans LA, USA, pp. 108 - 111, 11-13 Jan. 2010.

[5] D. Mardivirin, D. Bouyge, A. Crunteanu, A. Pothier, P. Blondy, "Study of Residual Charging in Dilectric Less Capacitive MEMS Switches", International Microwave Symposium, pp. 33-36, 2008

[6] U. Zaghloul, G. J. Papaioannou, H. Wang, B. Bhushan, F. Coccetti, P. Pons and R. Plana, "Nanoscale characterization of the dielectric charging phenomenon in PECVD silicon nitride thin films with various interfacial structures based on Kelvin probe force microscopy", Nanotechnology vol. 22, pp. 205708, 2011.

[7] U. Zaghloul, M. Koutsoureli, H. Wang, F. Coccetti, G. Papaioannou, P. Pons, R. Plana, "Assessment of dielectric charging in electrostatically driven MEMS devices: A comparison of available characterization techniques", Microelectronics Reliability 50, pp.1615-1620, 2010.

[8] M. S. Koutsoureli and G. J. Papaioannou, "Determination of bulk discharge current in the dielectric film of MEMS capacitive switches", Microelectronics Reliability, vol. 51, pp. 1874-1877, 2011.

[9] M. Koutsoureli and G. Papaioannou, "Determination of long time discharge current in microelectromechanical system capacitive switches", Applied Physics Letters 99, pp. 103503, 2011.

[10] D. Molinero and L. Castaner, "Dielectric charging characterization on microelectromechanical switches by discharge current transient", Applied Physics Letters 96, pp.183503, 2010.

[11] G. Papaioannou, "The Impact of Dielectric Material and Temperature on Dielectric Charging in RF MEMS Capacitive Switches" in NATO Science for Peace and Security Series - B: Physics and Biophysics, E. Gusev, E. Gurfankel and A. Dideikin Eds., Springer, 2009, pp. 141-153

[12] G. Papaioannou, "Dielectric Charging" in Advanced RF MEMS, S. Lucyszyn Ed., Cambridge University Press, pp.140-187, 2010.

[13] M. Koutsoureli, L. Michalas and G. Papaioannou, "The effect of temperature on dielectric charging of capacitive MEMS", 2011 IEEE International Reliability Physics Symposium, pp. 3D.4.1 - 3D.4.7, Monterey CA, 2011.

[14] X. Rottenberg, I. De Wolf, B. K. J. C. Nauwelaers, W. De Raedt and H. A. C. Tilmans, "Analytical Model of the DC Actuation of Electrostatic MEMS Devices With Distributed Dielectric Charging and Nonplanar Electrodes", J. of Microelectromechanical Systems, vol. 16, No.5, pp. 1243-1253, 2007.

[15] M. Pollak, "A percolation treatment of DC hopping conduction", Journal of Non Crystalline Solids 11, pp. 1-24, 1972.

[16] V. I. Arkhipov, E. V. Emelianova and G. J. Adriaenssens, "Field dependent effective temperature and variable range hopping: Application to dark dc conductivity in doped a-Si:H", Journal of Applied Physics, Vol. 93, No. 10, 2003.

[17] B. I. Shklovskii, "Hopping conduction in semiconductors in a strong electric field”, Sov. Phys. Semicond. Vol. 6, pp. 1964, 1973.

[18] M. Pollak and I. Riess, "A percolation treatment of high field hopping transport", Journal of Physics C: Solid State Physics, Vol. 9, pp. 23392352, 1976.

[19] U. Zaghloul, B. Bhushan, P. Pons, G. J. Papaioannou, F. Coccetti and R. Plana, "On the influence of environment gases, relative humidity and gas purification on dielectric charging/discharging processes in electrostatically driven MEMS/NEMS devices", Nanotechnology 22, pp. 035705, 2011.

[20] S. M. Sze, "Current transport and maximum dielectric strength of Silicon Nitride films", Journal of Applied Physics, vol. 38, No 7, pp. 2951 - 2956, 1967.

[21] K. J. B. M. Nieuwesteeg, A. A. van der Put, M. T. Johnson and C. G. C. de Kort, "DC bias stress of nonstoichiometric amorphous silicon nitride thin film diodes", Journal of Applied Physics, vol. 79, No. 2, pp. 842$849,1996$.

[22] M. Ben-Chorin, Z. Ovadyahu and M. Pollak, "Nonequilibrium transport and slow relaxation in hopping conductivity", Physical Review B, Vol. 48, No. 20, pp. 15025 - 15034, 1993.

[23] M. Koutsoureli, E. Papandreou, L. Michalas and G. Papaioannou, "Investigation of silicon nitride charging", Microelectronic Engineering 90, pp. 145-148, 2012.

[24] L. V. Govor, K. Boehme and J. Parisi, "Electrical field dependence of hopping conduction in self-organized carbon networks", Journal of Applied Physics, Vol. 91, No. 2, pp. 739-747, 2002. 